\title{
Metabolic Syndrome Prevalence in Renal Cell Cancer Patients
}

\author{
Suleyman Bulut, Binhan Kagan Aktas*, Akif Ersoy Erkmen, Cuneyt Ozden, \\ Cevdet Serkan Gokkaya, Mehmet Murat Baykam, Ali Memis
}

\begin{abstract}
Purpose: Renal cell carcinoma (RCC) is increasingly being recognized as a metabolic disease in recent studies. The aim of the present study was to identify the prevalence of metabolic syndrome (MetS) and its association with RCC among urologic patients. Materials and Methods: The study included a total of 355 participants (117 adult RCC patients and 238 age matched controls) divided into groups, with and without MetS diagnosed using the criteria of the American Heart Association/The National Heart Lung and Blood Institute. Groups were compared statistically and logistic regression analysis was performed to investigate the impact of MetS criteria on RCC risk. Results: Of the 117 RCC patients, 52 (44.4\%) and of the 238 controls, $37(15.5 \%)$ had MetS. A significant association $(p<0.001)$ was found between the presence of MetS and RCC (OR: 4.35; 95\% CI=2.627.21). As the number of MetS components accumulated from 3 to 5, RCC risk increased likewise from 4 to 6 times. Conclusions: MetS is more prevalent in RCC patients in Turkey compared to controls. Risk increases with the number of coexisting MetS components.
\end{abstract}

Keywords: Metabolic syndrome X - renal cancer - risk factor - Turkey

Asian Pac J Cancer Prev, 15 (18), 7925-7928

\section{Introduction}

Renal cell carcinoma (RCC) is the most common renal neoplasia, and it remains a very aggressive and often fatal disease (Protzel et al., 2012). It accounts for $2 \%$ of all adult malignancies (Jemal et al., 2010). About 270,000 new cases are diagnosed and 116,000 patients die of RCC per year worldwide (Ferlay et al., 2008).

In addition to genetic changes and cigarette smoking; obesity and hypertension (HT) have been shown to be significant metabolic risk factors for the development of RCC (Navai and Wood, 2012). Together with fasting hyperglycemia and abnormal serum lipid profile, obesity and HT are main components of a cluster of disorders known as metabolic syndrome (MetS). According to the definition of American Heart Association/The National Heart Lung and Blood Institute (AHA/NHLBI), which is the widely accepted one of numerous MetS definitions, the presence of any three or more of the following five risk factors has been defined as MetS: high waist circumference (WC), blood pressure (BP), fasting blood glucose (FBG), triglyceride (TG), and low high density lipoprotein-cholesterol (HDL-C) levels (Grundy et al., 2005).

MetS is very common in developed countries, and its prevalence is likely to increase (Ford et al., 2002). Several mechanisms have been proposed to explaine the relationship between MetS and malignant disease, including changes in neoplastic metabolism, DNA damage/repair malfunction, local inflammation and insulin-like growth factor 1 (Guo et al., 2013; Ozbek et al., 2014). It has been linked to certain types of cancer including breast cancer (Ronco et al., 2012), biliary tract cancer (Wu et al., 2012), colorectal cancer (Morita et al., 2005; Kaneko et al., 2010; Forootan et al., 2012; Ulaganathan et al., 2012) and aggressive urologic cancers involving prostate (Hammarsten and Peeker, 2011; Ozbek et al., 2014) and bladder (Ozbek et al., 2014). However, less is known about the possible link between MetS and RCC. Two of MetS components (HT and abdominal obesity) are known to cause RCC but it is not clear if MetS components completement each other to promote cancer. The cooperative effect of coexisted MetS components might go beyond our expectations. In the present casecontrol study, we aimed to identify the prevalence of MetS among RCC patients and to evaluate whether there is any association between the MetS and RCC.

\section{Materials and Methods}

At the urology clinic of our tertiary care teaching hospital, we designed a study in which 117 adult RCC patients and 238 age-matched ( \pm 2 years) controls were included between July 2010 and June 2013. RCC diagnose was based on histopathology reports of our radical or partial nephrectomies. Histologic subtype and nuclear grade of RCC were determined according to 2004 World Health Organization classification and Fuhrman grading 
system, respectively. Control group consisted of adult urologic patients attending our out-patient clinic with any complaints within the study period and agreeing to participate in the study. Patients with a known urinary or any other system cancer history were not included. All control group candidates were referred to ultrasonic evaluation and the subjects without a renal mass except simple renal cysts were included in the study. All participants were fully informed about the study design and their consent forms were obtained. The study was approved by applicable local ethics committee.

All patients and controls underwent BP and anthropometric measurements including weight, height, and WC; and body mass index (BMI) values were calculated accordingly. Physical measurements were made by the same observer (A.E.E.) using the same methods and instruments for all subjects. WC was measured at the midpoint of the lowest rib and iliac crest at the end of exhalation using a tape measure with the participant standing. BP was measured using Braun BP6200 Exactfit 5 Automatic Blood Pressure Monitor (Kaz Inc., Braun $\mathrm{GmbH}$, Kronberg, Germany). Blood samples were drawn from overnight-fasting subjects and serum levels of FBG, HDL-C and TG were recorded. Data of RCC patients were collected in the preoperative period. Criteria proposed for clinical diagnosis of MetS were provided by the report of AHA/NHLBI, any three of the five following factors: WC $\geq 102 \mathrm{~cm}$ in men and $\geq 88 \mathrm{~cm}$ in women; $\mathrm{TG} \geq 150 \mathrm{mg} / \mathrm{dL}$ or being on drug treatment for elevated TG; HDL-C $<40$ $\mathrm{mg} / \mathrm{dL}$ or being on drug treatment for reduced HDL-C; increased BP (systolic BP $\geq 130$ or diastolic $\mathrm{BP} \geq 85$ $\mathrm{mmHg}$ ) or being on antihypertensive drug treatment of previously diagnosed HT; increased FBG $\geq 100 \mathrm{mg} / \mathrm{dL}$ or being on drug treatment for elevated glucose (Grundy et al., 2005).

\section{Statistical analysis}

Statistical package for social sciences (SPSS, Chicago, IL, USA) version 21.0 software was used for the analysis of data. The data were normally distributed and expressed as the mean \pm standard deviation. The comparisons were performed by independent t-test, Fisher's exact and chisquare tests. Odds ratio (OR) was determined by binary logistic regression analysis to investigate the strength of the impact of MS criteria on RCC. P values $<0.05$ were considered significant.

At the end of the study, we conducted a post hoc power analysis with the program G*Power 3.1.9.2 software (Heinrich-Heine University, Dusseldorf, Germany) to identify the power achieved (Faul et al., 2009).

\section{Results}

Among a cohort of 355 subjects analyzed, the mean age was $57.36 \pm 10.43$ years. Of the cohort, $59.1 \%$ were male and $40.9 \%$ were female giving a male:female ratio of 1.4:1. Controls and RCC patients were well matched in age $(\mathrm{p}=0.688)$ as shown in table 1 . Significant differences were detected between control and $\mathrm{RCC}$ groups regarding mean levels of WC, TG and HDL-C but not regarding mean FBG and BMI (Table 1).
The prevalence of MetS as defined by AHA/NHLBI was $44.4 \%$ among RCC patients and $15.5 \%$ among controls. The prevalence was higher among males in both RCC and control groups (61.5\% and 56.8\%, respectively), compared to females (38.5\% and $43.2 \%$, respectively).

RCC is significantly $(\mathrm{p}=0.003)$ more prevalent in males than females (70.1\% and $29.9 \%$, respectively) and male gender 2.01 times increased the risk of RCC $(95 \%$ confidence interval $(\mathrm{CI})=1.26-3.22)$. HT had the most profound impact on RCC risk among MetS components because the presence of HT increased RCC risk more than 10 times (95\% CI=5.95-18.41) (Table 2).

We found a significant association $(\mathrm{p}<0.001)$ between the presence of MetS (having 3 or more MetS components) and $\mathrm{RCC}$ risk (OR: 4.35 ; 95\% CI=2.62-7.21). Besides, as the number of MetS components cumulated from 3 to 5, RCC risk increased likewise from 4 to 6 times (Table 3).

Table 1. Comparison of Mean Levels of Clinical Characteristics and Laboratory Findings of RCC Patients and Controls

\begin{tabular}{lrrrr}
\hline Characteristic & \multicolumn{1}{c}{ Control } & \multicolumn{1}{c}{ RCC } & $\mathrm{t}$ & $\mathrm{p}$ \\
\hline Patients & $238(67.0 \%)$ & $117(33.0 \%)$ & & \\
Age $(\mathrm{yr})$ & $57.53 \pm 9.35$ & $57.01 \pm 12.37$ & 0.402 & 0.688 \\
Height $(\mathrm{cm})$ & $168.27 \pm 7.59$ & $166.67 \pm 9.04$ & 1.656 & 0.099 \\
Weight $(\mathrm{kg})$ & $76.29 \pm 12.20$ & $76.57 \pm 12.27$ & -0.202 & 0.840 \\
BMI $\left(\mathrm{kg} / \mathrm{m}^{2}\right)$ & $26.98 \pm 4.28$ & $27.72 \pm 4.99$ & -1.369 & 0.173 \\
WC $(\mathrm{cm})$ & $95.57 \pm 10.26$ & $99.99 \pm 14.27$ & -2.995 & 0.003 \\
FBG $(\mathrm{mg} / \mathrm{dL})$ & $114.43 \pm 46.51$ & $118.98 \pm 52.84$ & -0.733 & 0.464 \\
TG $(\mathrm{mg} / \mathrm{dL})$ & $165.89 \pm 84.12$ & $143.53 \pm 68.20$ & 2.262 & 0.025 \\
HDL-C $(\mathrm{mg} / \mathrm{dL})$ & $45.25 \pm 12.84$ & $41.69 \pm 10.55$ & 2.455 & 0.015 \\
\hline
\end{tabular}

Table 2. Prevalence of Variables and their Associaton with RCC Risk

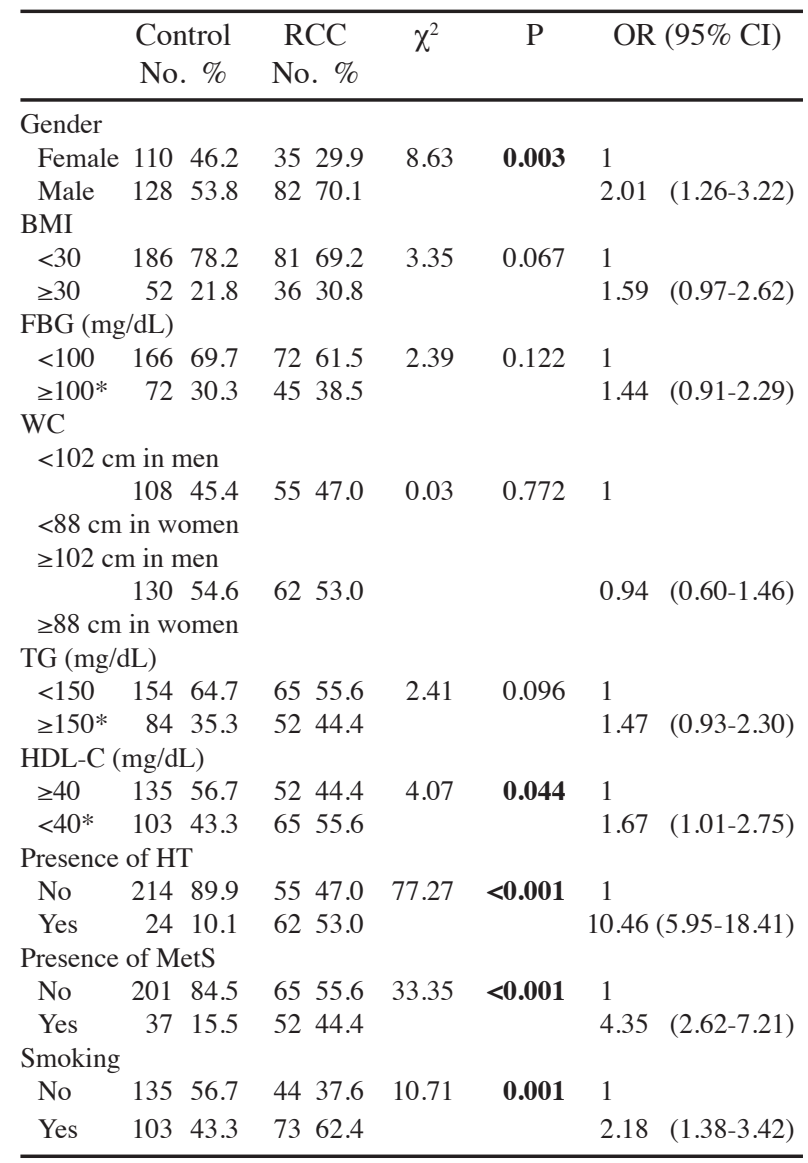

*or being on drug treatment 
Table 3. Prevalence of Number of MetS Components and its Association with RCC Risk

\begin{tabular}{|c|c|c|c|c|c|}
\hline $\begin{array}{l}\text { No. of MetS } \\
\text { components }\end{array}$ & $\begin{array}{l}\text { Control } \\
\text { No. } \%\end{array}$ & $\begin{array}{c}\text { RCC } \\
\text { No. } \%\end{array}$ & $\chi^{2}$ & $\mathrm{P}$ & OR $(95 \% \mathrm{CI})$ \\
\hline \multicolumn{6}{|c|}{$0-1-2($ not MetS) } \\
\hline & 20184.5 & 6555.6 & 35.25 & $<0.001$ & 1 \\
\hline 3 & 2610.9 & 3429.1 & & & $4.04(2.26-7.24)$ \\
\hline 4 & $8 \quad 3.4$ & 1210.3 & & & $4.64(1.82-11.84)$ \\
\hline$\underline{5}$ & 1.3 & $\begin{array}{ll}6 & 5.1 \\
\end{array}$ & & & $6.18(1.50-25.43)$ \\
\hline
\end{tabular}

Out of 117 RCC patients, $93(79.5 \%)$ were reported to have clear cell, $10(8.6 \%)$ papillary, $8(6.8 \%)$ chromophobe and $6(5.1 \%)$ other subtypes of RCC after the histopathological analyses. Fuhrman grading of RCC patients with and without MetS were as follows, respectively: grade 1 [(8 of $52(15.4 \%)$ and 10 of 65 $(15.4 \%)$, grade 2 [(27 of $52(51.9 \%)$ and 35 of 65 $(53.8 \%)$, grade 3 [(13 of $52(25.0 \%)$ and 16 of 65 $(24.6 \%)$ ], and grade 4 [(4 of $52(7.7 \%)$ and 4 of $65(6.2 \%)]$. Neither histological subtype nor nuclear grade of RCC was detected significantly different as to the presence or absence of MetS ( $\mathrm{p}=0.46$ and 0.99 , respectively).

Power of the present study to detect a medium effect size $(0.50)$ was calculated to be $99.3 \%$; critical $t=1.97$; $\mathrm{Df}=353$; noncentrality parameter $\delta=4.43$ at a significance level of 0.05 (two tailed) using t tests and to be $96.5 \%$; critical $\chi^{2}=11.07 ; \mathrm{Df}=5$; noncentrality parameter $\lambda=21.42$ using chi-square tests for a medium effect size (0.30) at the same significance level of 0.05 .

\section{Discussion}

$\mathrm{RCC}$ is increasingly being recognized as a metabolic disease. Each of the known genes for RCC is involved in oxygen, iron, and energy or nutrient-sensing pathways in a way or another (Azeem et al., 2011; Jonasch et al., 2012; Linehan and Ricketts, 2013). In a recent epidemiologic study from Northern Europe, Haggstrom et al. found that high levels of several metabolic factors, both separately and combined, were associated with an increased risk of RCC. Those metabolic factors were high levels of BMI, $\mathrm{BP}$, glucose, TG among men and BMI among women. However, the authors observed no interaction between the risk factors and RCC (Haggstrom et al., 2013). In the present study, we investigated the relationship between the risk of RCC and MetS which is referred to a constellation of interrelated metabolic risk factors. According to our results, MetS was highly prevalent based upon AHA/NHLBI criteria among RCC patients. For MetS assessment, the rationale for using AHA/NHLBI definition instead of another widely used definition of International Diabetes Federation (IDF), relied on the avoidance of a bias. Abdominal obesity is the sine qua non criterion for MetS definition of IDF (Alberti et al., 2005). Since obesity is a well-known risk factor for RCC, we have considered that it might constitude a bias if we used that definition, so we decided to use AHA/NHLBI criteria which are simple to use in a clinical setting and have the advantage of avoiding emphasis on a single cause (Grundy et al., 2005).

In the present study, it was found that the existence of MetS, about 4 fold increased the risk for RCC. MetS
DOI:http://dx.doi.org/10.7314/APJCP.2014.15.18. 7925 Metabolic Syndrome Prevalence in Renal Cell Cancer Patients alone was a significant risk intensive entity for RCC in our study cohort. The increase in RCC risk related to MetS was higher than other established RCC risk factors such as obesity and smoking. In addition, since obesity is a risk factor for RCC, our initial expectation was the mean weight and BMI of RCC patients were higher than controls. However, our RCC and control groups were not statistically different regarding BMI or total body weight, but there was a significant difference regarding WC. Therefore, we believe that WC was a better surrogate of obesity than BMI for our study population. Nevertheless, elevated WC did not change RCC risk in the present study after all. So, according to our results, it can be speculated that elevated WC or central obesity might have an impact on RCC risk when it only clusters with other MetS components.

As an interesting finding, there was a cumulative effect of MetS components for RCC risk in our study. When the number of MetS criteria combined from 3 to 5, RCC risk further increased up to 6.2 fold. This result might be interpreted reversely as a decrease in the number of MetS components would result in a reduced risk of RCC for MetS patients. For a preventive strategy for RCC, our practice should be trying to reduce not only the prevalence of MetS but also the number of its components.

The MetS (Ozbek et al., 2013) and diabetes mellitus itself (Otunctemur et al., 2014) have been shown to be associated with aggressive RCC in terms of higher tumor size and nuclear (Fuhrman) grade in recent studies that performed also in Turkish population. Their results point out a possible association between MetS and tumor carsinogenesis, eventhough we did not confirm any link either between MetS and nuclear grade or between MetS and RCC subtype. In another study, increased visceral obesity, a key component of MetS, was found to be strongly associated with higher Fuhrman grade in patients with clinical T1a RCC (Zhu et al., 2013). Since our results reflect the experience of a single institution, our study cohort may not have analogous regional or individual risk factors contributing to tumor aggressiveness or subtype formation with abovementioned studies. Therefore larger studies are needed to comment on this subject.

HT is another component of MetS, which was demonstrated to play an important role in the pathogenesis of RCC (Weikert et al., 2008). The highest incidence rate of moderate new-onset $\mathrm{HT}$ was observed in patients with RCC among various cancer types in a recent study (Fraeman et al., 2013). Brock et al demonstrated that both obesity and HT were independently and interactively associated with increased RCC risk (Brock et al., 2007). HT was found as the prominent MetS component for RCC risk in the present study. Van Hemelrijck et al. investigated the link between lipid profiles, glucose, BMI and kidney cancer in a large Swedish cohort, and showed a consistent relation between TG, glucose levels and kidney cancer (Van Hemelrijck et al., 2012). Our study only partially confirmed their results, because subjects with reduced HDL-C had significant risk for RCC whereas diabetics and subjects with hypertrigliseridemia had marginal, increased but statistically non-significant risk in our Turkish cohort.

One by one or in different pairings, particular MetS 
components have been found to increase RCC risk as mentioned above. However, the role of different combinations of existed MetS components for RCC risk, remains as an open question. This question might be answered by a large study identifying more risky combinations via comparing the patients under different subgroups of MetS components. However, it is hard to design such a study which has lots of risk subgroups. Instead of doing this, we believe that it had better assess the role of combination of risk factors for RCC under the joint term of MetS and investigate their interaction with each other.

We should acknowledge the limitations of our study. The results of our cohort should be corroborated in larger multicenter studies. Exposure to uncontrolled other nutritional or lifestyle factors that may confound the relationship between lifestyle and RCC, was another limitation. The treatment effect which was not a focus of our study, can be evaluated in future studies because treatment might change the MetS status of RCC patients.

Based on the current evidence, reductions in the prevalence of cigarette smoking, overweight and HT are preventive strategies for RCC (Weikert et al., 2010). Authors of the present study believe that MetS itself might be an established risk factor for RCC in the future with support of studies describing the exact causative underlying mechanism.

In conclusion, we found MetS more prevalent in a cohort of Turkish RCC patients compared to control group consisting of cancer-free urologic patients. The risk of RCC increased with the presence and the number of coexisting MetS components. From a public health point of view, urologists need to be aware of the effect that MetS and its components on RCC risk and should transfer this knowledge to their patients.

\section{References}

Alberti KG,Zimmet P, Shaw J (2005). IDF Epidemiology task force consensus group. the metabolic syndrome-a new worldwide definition. Lancet, 366, 1059-62.

Azeem K, Kollarova H, Horakova D, Magnuskova S, Janout V (2011). Genetic syndromes associated with renal cell carcinoma: a review. Biomed Pap Med Fac Univ Palacky Olomouc Czech Repub, 155, 231-8.

Brock K, Gridley G, Lynch C, Ershow A, Cantor K (2007). Obesity and hypertension interact to increase risk of renal cell carcinoma in Iowa, USA. Obes Res Clin Pract, 1, 1-2.

Faul F, Erdfelder E, Buchner A, Lang AG (2009). Statistical power analyses using $\mathrm{G}^{*}$ Power 3.1: tests for correlation and regression analyses. Behav Res Methods, 41, 1149-60.

Ferlay J, Shin HR, Bray F, et al (2008). Estimates of worldwide burden of cancer in 2008: GLOBOCAN. Int J Cancer, 127, 2893-917.

Ford ES, Giles WH, Dietz WH (2002). Prevalence of the metabolic syndrome among US adults: findings from the third national health and nutrition examination survey. JAMA, 287, 356-9.

Forootan M, Tabatabaeefar M, Yahyaei M, Maghsoodi N (2012). Metabolic syndrome and colorectal cancer: a cross-sectional survey. Asian Pac J Cancer Prev, 13, 4999-5002.

Fraeman KH, Nordstrom BL, Luo W, Landis SH, Shantakumar S (2013). Incidence of new-onset hypertension in cancer patients, a retrospective cohort study. Int J Hypertens, 2013, 379252.

Grundy SM, Cleeman JI, Daniels SR, et al (2005). American heart association, national heart, lung, and blood institute. diagnosis and management of the metabolic syndrome, an American heart association/national heart, lung and blood institute scientific statement. Circulation, 112, 2735-52.

Guo YZ, Pan L, Du CJ, Ren DQ, Xie XM (2013). Association between $\mathrm{C}$-reactive protein and risk of cancer: a metaanalysis of prospective cohort studies. Asian Pac J Cancer Prev, 14, 243-8.

Haggstrom C, Rapp K, Stocks T, et al (2013). Metabolic factors associated with risk of renal cell carcinoma. PLoS One, 8 , 57475 .

Hammarsten J, Peeker R (2011). Urological aspects of the metabolic syndrome. Nat Rev Urol, 8, 483-94.

Jemal A, Siegel R, Xu J, Ward E (2010). Cancer statistics. CA Cancer J Clin, 60, 277-300.

Jonasch E, Futreal PA, Davis IJ, et al (2012). State of the science, an update on renal cell carcinoma. Mol Cancer Res, 10,859-80.

Kaneko R, Sato Y, An Y, et al (2010). Clinico-epidemiologic study of the metabolic syndrome and lifestyle factors associated with the risk of colon adenoma and adenocarcinoma. Asian Pac J Cancer Prev, 11, 975-83.

Linehan WM, Ricketts CJ (2013). The metabolic basis of kidney cancer. Semin Cancer Biol, 23, 46-55.

Morita T, Tabata S, Mineshita M, et al (2005). The metabolic syndrome is associated with increased risk of colorectal adenoma development, the self-defense forces health study. Asian Pac J Cancer Prev, 6, 485-9.

Navai N, Wood CG (2012). Environmental and modifiable risk factors in renal cell carcinoma. Urol Oncol, 30, 220-4.

Otunctemur A, Ozbek E, Sahin S, et al (2014). Diabetes mellitus as a risk factor for high grade renal cell carcinoma. Asian Pac J Cancer Prev, 15, 3993-6.

Ozbek E, Otunctemur A, Sahin S, et al (2013). Renal cell carcinoma is more aggressive in Turkish patients with the metabolic syndrome. Asian Pac J Cancer Prev, 14, 7351-4.

Ozbek E, Otunctemur A, Dursun M, et al (2014). Association between the metabolic syndrome and high tumor grade and stage of primary urothelial cell carcinoma of the bladder. Asian Pac J Cancer Prev, 15, 1447-51.

Ozbek E, Otunctemur A, Dursun M, et al (2014). The metabolic syndrome is associated with more aggressive prostate cancer. Asian Pac J Cancer Prev, 15, 4029-32.

Pothiwala P, Jain SK, Yaturu S (2009). Metabolic syndrome and cancer. Metab Syndr Relat Disord, 7, 279-88.

Protzel C, Maruschke M, Hakenberg OW (2012). Epidemiology, aetiology and pathogenesis of renal cell carcinoma. Eur Urol Supp, 11, 52-9.

Ronco AL, De Stefani E, Deneo-Pellegrini H, Quarneti A (2012). Diabetes, overweight and risk of postmenopausal breast cancer, a case-control study in Uruguay. Asian Pac J Cancer Prev, 13, 139-46.

Ulaganathan V, Kandiah M, Zalilah MS, et al (2012). Colorectal cancer and its association with the metabolic syndrome, a Malaysian multi-centric case-control study. Asian Pac J Cancer Prev, 13, 3873-7.

Van Hemelrijck M, Garmo H, Hammar N, et al (2012). The interplay between lipid profiles, glucose, BMI and risk of kidney cancer in the Swedish AMORIS study. Int J Cancer, 130, 2118-28.

Weikert S, Boeing H, Pischon T, et al. (2008). Blood pressure and risk of renal cell carcinoma in the European prospective investigation into cancer and nutrition. Am J Epidemiol, 167, 438-46.

Weikert S, Ljungberg B (2010). Contemporary epidemiology of renal cell carcinoma, perspectives of primary prevention. World J Urol, 28, 247-52.

Wu Q, He XD, Yu L, Liu W, Tao LY (2012). The metabolic syndrome and risk factors for biliary tract cancer, a case-control study in China. Asian Pac J Cancer Prev, 13, 1963-9.

Zhu Y, Wang HK, Zhang HL, et al (2013). Visceral obesity and risk of high grade disease in clinical t1 a renal cell carcinoma. J Urol, 189, 447-53. 